\title{
Effets de l'incorporation de graines de lin floconnées sur support d'orge chez le taurillon à l'engraissement *, **
}

\author{
I Dufrasne, M Gielen, L Istasse, C Van Eenaeme, A Gabriel, \\ A Clinquart, JM Bienfait
}

\begin{abstract}
Faculté de médecine vétérinaire, service de nutrition 45 , rue des Vétérinaires, 1070 Bruxelles, Belgique
\end{abstract}

(Reçu le 24 avril 1990; accepté le 10 octobre 1990)

\begin{abstract}
Résumé - Des graines de lin floconnées sur support d'orge ont été utilisées dans une ration pour taurillons à l'engraissement. La ration était à base de pulpes séchées et complémentée par de l'orge, du tourteau de soja et des graines de lin floconnées qui représentaient $10 \%$ des apports. Dans la ration témoin, du tourteau de lin a été utilisé de manière à apporter une quantité de protéine brute semblable à celle apportée par les graines de lin.

L'incorporation de graines de lin a augmenté la teneur en extrait éthéré de 2,1 à $4,1 \%$. La durée d'engraissement a été plus longue de $5 \mathrm{j}$ chez les animaux complémentés en matière grasse. Le gain a été supérieur de $18 \mathrm{~kg}(P<0,05)$, ce qui correspondait à des gains quotidiens moyens de $1,62 \mathrm{~kg}$ contre $1,44 \mathrm{~kg}$ pour la ration témoin. Les consommations ont été légèrement inférieures dans le lot complémenté. Les carcasses ont été plus grasses $(P<0,10)$ et la graisse périrénale des animaux qui ont reçu la ration supplémentée contenait $2 \%$ d'acide linolénique de plus $(P<0,05)$. II n'y a pas eu de différences significatives entre régimes au niveau des métabolites sanguins.

La dégradabilité dans le rumen des graines de lin floconnées sur support d'orge, mesurée par la technique des sachets de nylon, s'est révélée élevée : environ $40 \%$ après $2 \mathrm{~h}$ d'incubation et plus de $78 \%$ après $24 \mathrm{~h}$. Par contre, il semble que l'introduction de graines de lin floconnées dans la ration ait permis un approvisionnement plus élevé en acides gras polyinsaturés à la sortie du rumen, comme l'indiquent les teneurs élevées en ces acides gras polyinsaturés dans les résidus et dans la graisse animale.
\end{abstract}

engraissement taurillon / graine de lin floconnée / performance zootechnique / acide gras polyinsaturé

Summary - Effects of steam treated linseed with $\mathrm{h} . . \quad$ support on the performances of growing fattening bulls. Steam treater for growing fattening bulls. The dic ... $\quad \ldots$ on sugar beet pulp and was supplemented with barley, soyabean meal and steam : .... . iilseed at an incorporation rate of $10 \%$. In the control diet, linseed meal was used so that the amount of crude protein was similar to that as with the linseed.

The incorporation of linseed increased the ether extract content of the diet (4.1 vs $2.1 \%)$. the length of the fattening period was $5 \mathrm{~d}$ longer with the bulls supplemented with fat. Similarly, the total live weight gain was $18 \mathrm{~kg}(\mathrm{P}<0,05)$ higher than with the control diet, corresponding to an average daily gain of $1620 \mathrm{~g}$ as compared with $1440 \mathrm{~g}(\mathrm{P}<0,20)$.

Feed intake was slightly reduced with the fat supplemented diet. The carcasses were fatter: 20.8 vs $18.4 \%$ adipose tissue $(P<0,10)$ and the perirenal fat of the bulls with the fat supplemented diet

\footnotetext{
* Recherche réalisée avec la collaboration financière de l'institut pour l'encouragement de la recherche scientifique dans l'industrie et l'agriculture (IRSIA, Bruxelles, Belgique)

** Les graines de lin floconnées ont été produites par la firme Interagri (Seilles, Belgique)
} 
contained $2 \%$ more linolenic acid $(\mathrm{P}<0,05)$ that these of the bulls with the control diet. There were no significant differences between diets for plasma metabolites.

The digestibility in the rumen, measured by the nylon bag technique, of steam treated linseed with barley as support was high: about $40 \%$ after $2 h$ of incubation and over $78 \%$ after $24 h$. By contrast, it appeared that the incorporation of steam treated linseed in the diet increased the outflow of desaturated fatty acids from the rumen as indicated by the high content in desaturated fatty acids found in the residues and in animal fat.

Fattening bull / steam treated linseed / animal performance / polyinsatured fatty acid

\section{INTRODUCTION}

La technique de l'engraissement précoce du taurillon nécessite le recours à des régimes très denses en énergie. En Belgique, ces derniers sont à base de pulpes séchées de betteraves sucrières (Cordiez et al, 1977) ou à base d'ensilage de maïs. La teneur en matière grasse dans ces rations est généralement faible. Actuellement, les fabricants d'aliments pour animaux à l'engrais ont tendance à incorporer de plus en plus souvent des matières grasses. Ce procédé a pour but d'augmenter la densité en énergie de la ration et d'assurer un approvisionnement en acides gras à longue chaîne.

Le but du présent travail est d'étudier les effets de l'enrichissement d'une ration d'engraissement par des graines de lin floconnées sur support d'orge sur les performances des taurillons à l'engrais.

\section{MATÉRIEL ET MÉTHODES}

\section{Essai 1}

\section{Animaux}

Seize taurillons de race Blanc-Bleu Belge de type mixte ont été utilisés. Ces animaux avaient passé la saison de pâturage ensemble. A leur rentrée, en automne, ils ont été soumis à une période de transition de $15 \mathrm{j}$ et ensuite, ils sont passés en engraissement. Ils étaient âgés d'environ 15 mois et pesaient environ $420 \mathrm{~kg}$. Ils ont été répartis de façon homogène en 2 groupes de 8 animaux. Le premier groupe recevait la ration témoin tandis que le second recevait la ration enrichie en graines de lin floconnées sur support d'orge. Chaque groupe d'animaux était maintenu dans un box en stabulation libre semipaillée.

\section{Alimentation}

Les rations d'engraissement étaient à base de pulpes séchées (tableau I). Elles étaient complémentées en amidon par de l'orge. Les sources de matières azotées complémentaires étaient le tourteau de soja et le tourteau de lin dans la ration témoin. Des graines de lin floconnées ont été utilisées dans la ration expérimentale qui s'est trouvée de ce fait enrichie en matière grasse. Pour des raisons techniques, le floconnage a été réalisé sur un mélange contenant $50 \%$ de graines de lin et $50 \%$ d'orge. La température à la fin du processus de floconnage était de $100^{\circ} \mathrm{C}$. Les graines de lin floconnées ont été substituées au tourteau de lin et à une partie des pulpes séchées. La quantité de matières azotées apportées par les graines de lin correspondait à celle apportée par le tourteau de lin dans la ration témoin. Dans la ration enrichie en matière grasse, l'orge était présentée, en partie, sous forme floconnée et sous forme moulue, tandis qu'elle était présentée sous forme moulue dans la ration témoin. La distribution des aliments était proche de ad libitum par un ajustement régulier des quantités jusqu'à l'apparition de refus. La ration a été distribuée en 2 repas par jour, les animaux ayant accès en permanence à de la paille au ratelier. 
Tableau I. Composition des rations distribuées aux 2 groupes de taurillons à l'engrais.

\begin{tabular}{|c|c|c|}
\hline $\begin{array}{l}\text { Composition } \\
\text { des régimes }\end{array}$ & Témoin & $\begin{array}{l}\text { Pation } \\
\text { Supplément } \\
\text { en graines } \\
\text { de lin } \\
\text { floconnées }\end{array}$ \\
\hline \multicolumn{3}{|l|}{ Composition centésimale } \\
\hline Tourteau de soja & 10,0 & 10,0 \\
\hline Tourteau de lin & 6,0 & - \\
\hline Graine de lins floconnées & - & 10,0 \\
\hline Orge & 35,0 & 35,0 \\
\hline Pulpes séchées & 47,5 & 43,5 \\
\hline Mélange minéral & 1,5 & 1,5 \\
\hline \multicolumn{3}{|l|}{ Composition chimique } \\
\hline Cendres (\% MS) & 6,9 & 6,8 \\
\hline Fibres brutes (\% MS) & 12,5 & 11,8 \\
\hline UFV (/kg MS)* & 1,01 & 1,04 \\
\hline \multicolumn{3}{|l|}{ Matière azotée totale } \\
\hline$(\mathrm{N} \times 6,25)(\% \mathrm{MS})$ & 16,0 & 16,1 \\
\hline Extrait éthéré (\% MS) & 2,1 & 4,1 \\
\hline
\end{tabular}

* unité fourragère viande.

\section{Mesures}

Les quantités d'aliments ont été pesées quotidiennement pour chacun des 2 lots. Les taurillons ont été pesés tous les 28 j par doubles pesées. Quatre prélèvements sanguins ont été effectués à la veine jugulaire à 28 j d'intervalle, $4 \mathrm{~h}$ après la distribution des aliments du matin. Les animaux ont été abattus en 3 séries en fonction de leur état de finition. Ce dernier était déterminé par le chevillard grâce aux maniements. A l'abattage, les carcasses ont été pesées à chaud. Un segment monocostal a été prélevé au niveau de la $7 e$ côte, en vue d'estimer la composition de la carcasse (Michaux et al, 1983). Un échantillon de graisse périrénale a également été prélevé sur 5 taurillons de chaque lot afin de déterminer la composition en acides gras.

\section{Essai 2}

La mesure de la dégradabilité du mélange graines de lin et orge floconnées a été effectuée suivant la méthode des sachets de nylon décrite par Mehrez et Ørskov (1977). Les sachets, d'une taille de $12 \times 7,5 \mathrm{~cm}$ ont été cousus à partir d'une taille de nylon dont les mailles mesuraient $63 \mu \mathrm{m}$ de côté. Ils ont été remplis d'environ $6 \mathrm{~g}$ du mélange préalablement passé dans un moulin à marteaux muni d'un crible à mailles de $5 \mathrm{~mm}$ de diamètre. Les sachets ont été incubés pendant $2,4,8,16,24$ et $48 \mathrm{~h}$ dans le rumen de 3 boeufs qui recevaient une ration composée de $50 \%$ de foin et de $50 \%$ de concentré. Après incubation, les sachets ont été séchés, non pas à l'étuve comme décrit dans la technique originale, mais par lyophilisation, pour éviter la fusion des matières grasses. Les résidus secs de chaque lot (correspondant à un temps d'incubation) ont été mélangés, en vue d'être analysés pour déterminer les teneurs en matière grasse et en acides gras.

\section{Mesures}

La composition des aliments a été déterminée par analyse de Weende. La détermination de la composition en acides gras des échantillons de graisse périrénale, de graines de lin, de graines de lin floconnées et des résultats d'incubation a été réalisée par chromatographie gazeuse capillaire en appliquant la technique décrite par Ter Meulen et Molnar (1975) : homogéneisation et extraction par du chloroforme-méthanol (2 : 1), addition d'acide heptadécanoïque comme standard interne, évaporation du solvant, saponification dans du $\mathrm{KOH}$-méthanol à $15 \%$, séparation de la fraction insaponifiable par extraction à l'éther de pétrole $\left(40-60^{\circ} \mathrm{C}\right)$, acidification de la phase aqueuse par de l' $\mathrm{HCl}(1: 3)$ et extraction des acides gras dans de l'éther de pétrole, estérification des acides par du BF3-méthanol et isolation des esters méthyliques dans de l'éther de pétrole. La séparation des esters a été réalisée sur une colonne capillaire en "fused silica" de $25 \mathrm{~m}$ de longueur, 0,32 $\mathrm{mm}$ de diamètre intérieur avec comme phase stationnaire du polysiloxane-cyanopropyle DB 225 (JxW); l'hydrogène a été utilisé comme gaz porteur. Le chromatographe utilisé était le modèle 427 de Packard Instruments, relié à un intégrateur-enregistreur Hewlett-Packard 3380A. L'échantilion était injecté en système "direct injection" dans un "pyrex insert" et la détection se faisait par un détecteur à ionisation de flamme. Le sang a été récolté dans des tubes $\mathrm{NaF}$-oxalate en vue de la déter- 
mination des concentrations en urée, azote $\alpha$ aminé, glucose, créatinine et des triglycérides. Les triglycérides ont été mesurés par la méthode GPO-PAP basée sur une hydrolyse enzymatique des triglycérides suivie du dosage en colorimétrie du glycérol libéré en version kit de Boehringer Mannheim GmbH (réf 701909). Les concentrations des 4 autres métabolites étaient déterminées par des méthodes colorimétriques automatisées à l'aide d'un Autoanalyzer Technicon : le glucose et l'urée respectivement par les méthodes à l'orthotoluidine et à la diacétylmonoxine (Henry et al, 1974), l'azote $\alpha$-aminé par la réaction au trinitrobenzène sulphonate en utillisant la méthode de Palmer et Peters (1969) et la créatinine par la méthode de Jaffe (Henry et al, 1974).

\section{Analyse statistique des résultats}

Le test de $t$ de student a été utilisé pour comparer les performances des animaux recevant la ration témoin et la ration enrichie en matière grasse.

\section{RESULTATS}

\section{Essai 1}

\section{Composition des aliments}

La composition chimique de l'orge, des pulpes séchées, du tourteau de lin et du tourteau de soja était classique. Les teneurs en fibres brutes, protéines et matière grasse du mélange graines de lin et orge floconnées étaient respectivement de $6,5,17,9$ et $14,4 \%$ dans la matière sèche (MS). Les teneurs dans la MS en matières azotées totales des rations, calculées à partir de la composition centésimale et de l'analyse des aliments étaient de 16,0 et $16,1 \%$ dans la ration témoin et la ration enrichie en matière grasse. Les teneurs en extrait éthéré étaient respectivement de 2,1 et $4,1 \%$.

\section{Performances des animaux}

Deux taurillons du groupe recevant la ration témoin ont été écartés au cours du premier mois à la suite de boiteries entraînées par des glissades. Le tableau II résume les performances zootechniques des 2 groupes de taurillons. Le poids moyen initial était de $416 \pm 13 \mathrm{~kg}$ dans le groupe témoin et de $422 \pm 43 \mathrm{~kg}$ dans le groupe complémenté en graines de lin floconnées. La durée d'engraissement des animaux qui ont reçu la ration enrichie en graines de lin n'a pas été significativement différente, bien que plus longue de $5 \mathrm{j}$. Le poids final a été supérieur de $24 \mathrm{~kg}$ avec la ration complémentée $(P<0,20)$ et le gain total a été majoré de $18,0 \mathrm{~kg}(P<0,05)$, ces augmentations étant en partie attribuables au séjour plus long des animaux. Le gain quotidien moyen a été plus élevé chez les taurillons nourris avec la ration complémentée : 1,62 vs $1,44 \mathrm{~kg} / \mathrm{j}(P<0,20)$. La consommation journalière et l'indice de consommation du groupe complémenté ont été inférieurs de $0,17 \mathrm{~kg}$ et $0,75 \mathrm{~kg}$ repectivement. Le poids d'abattage moyen a été supérieur de $28 \mathrm{~kg}(P<0,10)$, la freinte réduite de $0,8 \%$ et le poids des carcasses supérieur de $16 \mathrm{~kg}(P<0,10)$ chez les animaux qui ont reçu les graines de lin floconnées. Les rendements des carcasses, c'est-à-dire le rapport entre les poids de carcasse et d'abattage, ont été semblables dans les 2 groupes : 56,4 et $56,5 \%$. Le pourcentage de muscles a été supérieur chez les animaux qui ont reçu la ration témoin : 67,5 vs $66,1 \%$ tandis que le pourcentage de tissu conjonctivo-adipeux était inférieur : 18,4 vs $20,8 \%(P<0,10)$.

Les concentrations moyennes en triglycérides, créatinine, glucose, utée et azote $\alpha$-aminé déterminées sur les plasmas des sangs prélevés mensuellement sont données dans le tableau III. II n'y a pas eu de différence significative entre les 2 régimes 
Tableau II. Performances de taurillons en croissance-engraissement recevant une ration contenant des graines de lin floconnées (moyenne \pm écart type).

\begin{tabular}{|c|c|c|}
\hline $\begin{array}{l}\text { Variables mesurées } \\
\text { ou calculées }\end{array}$ & Témoin & $\begin{array}{l}\text { Supplément en graines } \\
\text { de lin floconnées }\end{array}$ \\
\hline Poids initial $(\mathrm{kg})$ & $416 \pm 13$ & $422 \pm 43$ \\
\hline Poids final $(\mathrm{kg})$ & $520 \pm 13$ & $544 \pm 36$ \\
\hline Durée d'engraissement (j) & $72 \pm 7$ & $77 \pm 14$ \\
\hline Gain quotidien $(\mathrm{kg})$ & $1,44 \pm 0,18$ & $1,62 \pm 0,30$ \\
\hline Consommation (kg/j) & $9,42 \pm 0,09$ & $9,25 \pm 0,40$ \\
\hline Indice de consommation ( $\mathrm{kg} / \mathrm{kq}$ ) & $6,65 \pm 0,80$ & $5,90 \pm 1,03$ \\
\hline Poids d'abattage $(\mathrm{kg})$ & $512 \pm 12$ & $540 \pm 36$ \\
\hline Freinte (\%) & $1,5 \pm 0,6$ & $0,7 \pm 0,4$ \\
\hline Poids de carcasse $(\mathrm{kg})$ & $289 \pm 11$ & $305 \pm 16$ \\
\hline Rendement (\%) & $56,4 \pm 1,1$ & $56,5 \pm 1,9$ \\
\hline Muscles dans carcasse (\%) & $67,5 \pm 2,1$ & $66,1 \pm 3,0$ \\
\hline Tissus adipeux dans carcasse (\%) & $18,4 \pm 1,9$ & $20,8 \pm 2,8$ \\
\hline
\end{tabular}

en ce qui concerne les métabolites mesurés.

La répartition des acides gras contenus dans la graisse périrénale des taurillons est donnée au tableau IV. L'acide oléique et l'acide palmitique ont été retrouvés dans des proportions plus élevées, bien que non significatives, chez les taureaux du groupe témoin : 36,66 vs 33,49 et 25,43 vs $24,52 \%$ respectivement. Les proportions d'acide stéarique et d'acide linoléique ont été plus élevées sans être significative- ment différentes chez les taurillons du groupe complémenté : 30,02 vs 29,07 et 3,63 vs $3,49 \%$ respectivement. La teneur en acide linolénique a été significativement plus élevée dans le lot complémenté : 2,48 vs $0,51 \%(P<0,05)$.

\section{Essai 2}

La teneur en extrait éthéré était de $14,4 \%$ dans la matière sèche du mélange conte-

Tableau III. Concentrations moyennes en métabolites dans le plasma sanguin.

\begin{tabular}{lcc}
\hline \multicolumn{1}{c}{ Métabolites } & Témoin & $\begin{array}{c}\text { Ration } \\
\text { Supplément en graines } \\
\text { de lin floconnées }\end{array}$ \\
\hline Triglycérides $(\mathrm{g} / \mathrm{l})$ & $0,142 \pm 0,002$ & $0,130 \pm 0,032$ \\
Créatinine $(\mathrm{mg} \mathrm{N} / \mathrm{l})$ & $15,8 \pm 0,3$ & $14,8 \pm 0,4$ \\
Glucose $(\mathrm{mg} / \mathrm{l})$ & $804,5 \pm 16,3$ & $802,0 \pm 15,4$ \\
Urée $(\mathrm{mg} \mathrm{N} / \mathrm{l})$ & $94,9 \pm 10,0$ & $83,5 \pm 5,3$ \\
Azote $\alpha$-aminé $(\mathrm{mg} \mathrm{N} / \mathrm{l})$ & $51,8 \pm 0,7$ & $52,5 \pm 1,4$ \\
\hline
\end{tabular}


Tableau IV. Répartition des acides gras dans la graisse périrénale.

\begin{tabular}{lrr}
\hline & \multicolumn{2}{c}{ Ration } \\
Acides gras & Témoin & $\begin{array}{l}\text { Supplément } \\
\text { en graines de } \\
\text { lin floconnées }\end{array}$ \\
\hline & & \\
C16:0 & $25,43 \pm 2,75$ & $24,52 \pm 3,77$ \\
C18:0 & $29,07 \pm 1,43$ & $30,02 \pm 2,27$ \\
C18:1 & $36,66 \pm 4,69$ & $33,49 \pm 3,13$ \\
C18:2 & $3,49 \pm 0,90$ & $3,63 \pm 2,20$ \\
C18:3 & $0,51 \pm 0,43$ & $2,48 \pm 1,63$ \\
C20:0 & $1,12 \pm 1,00$ & $2,22 \pm 1,99$ \\
C24:0 & $3,51 \pm 3,76$ & $3,63 \pm 4,45$ \\
\hline
\end{tabular}

nant les graines de lin floconnées et du résidu après $8 \mathrm{~h}$ d'incubation. La dégradabilité dans le rumen de la matière sèche et de l'extrait éthéré de graines de lin floconnées sur support d'orge est donnée dans le tableau V. La dégradabilité de l'extrait éthéré dans le résidu incubé pendant $48 \mathrm{~h}$ n'a pas été mesurée. Les dégradabilités ont été importantes puisqu'elles étaient déjà de 39,01 et $42,74 \%$ respectivement après $2 \mathrm{~h}$ d'incubation; elles se sont élevées à plus de $78 \%$ après $24 \mathrm{~h}$.

La figure 1 illustre la répartition des principaux acides gras contenus dans les graines de lin au cours de l'incubation dans le rumen.

Les graines de lin floconnées étaient, comme les graines de lin non traitées, riches en acide linolénique. Néanmoins, il faut noter une teneur légèrement inférieure en cet acide au profit de l'acide linoléique après le floconnage (45 vs $50 \%$ ). Au niveau des résidus d'incubation dans le rumen, une diminution assez sensible des teneurs en acides linolénique et linoléique a été observée au profit principalement des acides oléique, stéarique et palmiti-
Tableau V. Dégradabilité en $\%$ de la matière sèche et de l'extrait éthéré en fonction des temps d'incubation.

\begin{tabular}{ccc}
\hline \multirow{2}{*}{$\begin{array}{c}\text { Temps d'incubation } \\
(h)\end{array}$} & \multicolumn{2}{c}{ Dégradabilité (\%) } \\
\cline { 2 - 3 } & Matière sèche Extrait éthéré \\
\hline & & \\
2 & 39,01 & 42,74 \\
4 & 56,03 & 57,37 \\
8 & 70,73 & 69,12 \\
16 & 68,77 & 71,65 \\
24 & 78,82 & 85,86 \\
48 & 82,84 & - \\
\end{tabular}

que. La proportion d'acides gras polyinsaturés était de $67 \%$ dans les graines de lin floconnées; elle a chuté à $36 \%$ après 24 h d'incubation.

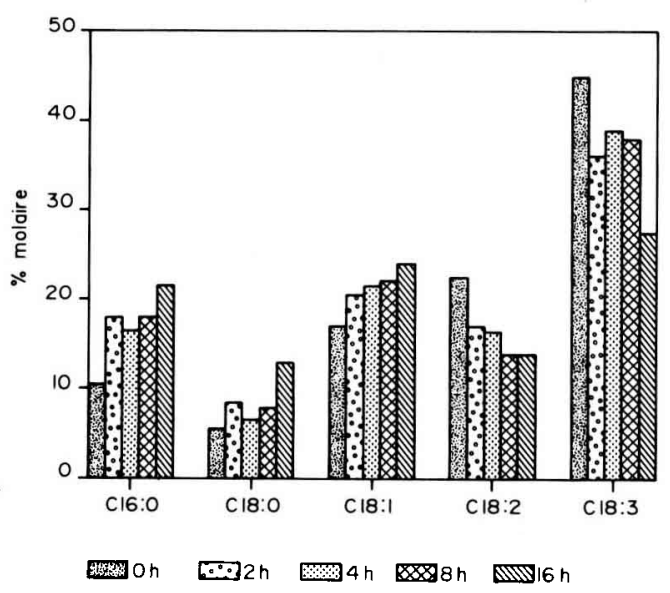

Fig 1. Répartition des principaux acides gras contenus dans les graines de lin floconnées au cours de l'incubation dans le rumen. 


\section{DISCUSSION}

La ration d'engraissement complémentée par des graines de lin floconnées sur support d'orge a permis d'obtenir une augmentation du gain quotidien de $12,5 \%$ et une réduction de l'indice de consommation de $11,3 \%$. L'amélioration des performances observées avec la supplémentation peut être le résultat de différents effets : augmentation de la teneur en matière grasse, apport en acides gras, augmentation de la teneur en énergie, amélioration de l'efficience de l'utilisation de la ration et protection des matières azotées, suite au floconnage. La littérature ne donne pas d'information sur des essais zootechniques en production de viande dans lesquels des graines de lin floconnées ont été utilisées. Par contre, il existe de nombreuses expériences où des matières grasses d'origine végétale ont été incorporées à des taux variables. L'effet d'une complémentation en matière grasse sur les performances animales dépend de différents facteurs : type de matière grasse, taux d'incorporation et autres composants de la ration. L'addition de 3,5\% d'huile de soja non protégée dans une ration pour taurillons à l'engraissement a augmenté significativement l'efficacité alimentaire (Brandt et Anderson, 1990). Une augmentation significative du gain et de l'indice de consommation a aussi été rapportée par Zinn (1989) après distribution de 4\% d'un mélange de graisses extraites de déchets de collectivités et de graisses animales. Dans un essai dose -effet, Chandler et al (1966) ont fait varier chez le veau le taux d'incorporation d'huile de maïs de 0 à $8 \%$ et celui du tourteau de soja de 0 à $28 \%$ et ont démontré que le gain était plus élevé avec $2 \%$ d'huile de maïs et $16 \%$ de tourteau de soja. En incorporant $5 \%$ d'huile de tournesol ou d'huile de soja dans des rations pour bouvillons, Roberts et Mc Kirdy
(1964) ont également noté une amélioration des gains et de l'efficacité alimentaire. Par contre, dans l'expérience de Willey et al (1957), l'incorporation de 5\% d'huile de coton n'a pas modifié les gains mais a amélioré l'efficacité alimentaire. L'incorporation de $15 \%$ d'huile de maïs dans une ration pour agneaux à l'engraissement n'a pas entrainé de modification de gain à condition que la ration contienne suffisamment de fibres (Cameron et Hogue, 1968). Une diminution de gain a été observée avec $10 \%$ d'huile de maïs dans des rations pour moutons par Brethour et al (1958). II semble donc que, comme pour la vache laitière, dans la plupart des rations, la complémentation avec 4 ou $8 \%$ d'acides gras à longue chaîne soit suffisante pour optimiser la productivité et la santé animale (Palmquist et Conrad, 1978; Chalupa et al, 1986). L'incorporation de lipides, et en particulier de matières grasses riches en acides gras polyinsaturés, doit être relativement limitée car un excès peut inhiber l'activité microbienne, surtout les microorganismes responsables de la cellulolyse (Czerkawski et al, 1975; lkwuegbu et Sutton, 1982; Zinn, 1988). En outre, l'effet sur la croissance microbienne dépend de la concentration en acides gras : effet stimulateur à basse concentration et effet inhibiteur à concentration élevée (llian et al, 1988). Finalement, l'amélioration de l'efficacité alimentaire peut être associée à l'approvisionnement direct en acides gras préformés au niveau tissulaire ou à la génération d'énergie à partir de l'oxydation des acides gras à longue chaîne (Palmquist, 1988).

Les poids d'abattage et de carcasse des taurillons complémentés ont été supérieurs, avec des rendements semblables. Des poids de carcasse significativement plus élevés ont été signalés par Brandt et Anderson (1990), ainsi qu'une augmentation significative du rendement. De même, 
une augmentation du poids d'abattage et de carcasse a été observée par Zinn (1989). L'huile de colza avait provoqué une réduction et l'huile de tournesol, une augmentation des rendements dans les travaux de Roberts et Mc Kirdy (1964).

Le pourcentage de tissu conjonctivoadipeux a été supérieur de $2 \%$ pour les taurillons complémentés. Zinn (1989) avait observé, chez le boeuf, une augmentation non significative du pourcentage de graisse dans la carcasse, ainsi qu'une augmentation significative des proportions de graisses pelvienne, cardiaque et rénale. Des résultats similaires ont été obtenus par Brandt et Anderson (1990). Bien que non déterminée dans l'expérience présente, il est vraisemblable qu'une partie de l'augmentation de la proportion de graisse provienne également d'un dépôt plus important dans les cavités pelvienne et abdominale.

La graisse périrénale des taurillons complémentés en graines de lin contenait un pourcentage significativement plus élevé d'acide linolénique. Différents auteurs ont rapporté que des rations riches en acides gras polyinsaturés, et plus particulièrement en acide linoléique, peuvent influencer la composition de la graisse déposée. Une incorporation de $6 \%$ d'huile de carthame a entraîné, chez le boeuf, une augmentation significative de l'acide linoléique des graisses intramusculaires (Dryden et Marchello, 1973). Garret et al (1976) ont observé une augmentation de la concentration en acide linoléique dans la matière grasse totale, après avoir distribué des lipides encapsulés dans un complexe formol protéine. Lassiter (1968) a montré que les concentrations en acides oléique et linoléique ont augmenté de façon significative lors de distribution, chez le mouton, de rations contenant $5 \%$ d'huile de maïs. Marchello et al (1969) ont obtenu également une augmentation en acide linoléique dans la graissse de mouton après avoir injecté $3 \%$ d'huile de maïs dans la caillette; l'augmentation a été moindre lorsque l'huile était incorporée dans la ration. De même, les incorporations d'huile de maïs de d'huile de tournesol réalisées par Cameron et Hogue (1968), Gibney et l'Estrange (1975) et Johnson et Mc Clure (1972) dans des rations pour moutons ont entraîné une augmentation de l'acide linoléique dans la graisse.

Après l'incubation dans le rumen, $36 \%$ d'acides gras polyinsaturés étaient encore présents. II semble donc qu'une large proportion des acides gras des graines de lin floconnées introduites dans la ration aient résisté aux biohydrogénations par les microorganismes, permettant ainsi un approvisionnement plus important en ces acides à l'entrée du duodénum. Une autre origine des matières grasses dans les résidus d'incubation consiste en la synthèse de novo d'acides gras par les microorganismes (Harfoot, 1981). D'autre part, d'après Bauchart et al (1990), l'incorporation des acides gras dans les bactéries constituerait un moyen de protection des acides gras polyinsaturés contre la biohydrogénation.

De la présente expérience, et mise à part une augmentation de la proportion de graisse dans la carcasse, il ressort que l'utilisation de graines de lin floconnées a amélioré les performances zootechniques de taurillons à l'engrais. Une partie des acides gras contenus dans les graines de lin floconnées semble avoir échappé à l'hydrogénation par les microorganismes du rumen et atteint le duodénum comme le suggère le pourcentage élevé d'acides gras polyinsaturés encore présents après incubation et l'augmentation de ces acides gras dans la graisse des animaux. 


\section{RÉFÉRENCES}

Bauchart D, Legay-Carmier F, Doreau $M$, Gaillard B (1990) Lipid metabolism of liquid associated and solid adherent bacteria in rumen content of dairy cows offered lipid supplemented diets. Br J Nutr 63, 563-578

Brandt JR, Anderson SJ (1990) Supplemental fat affects feedlot performances and carcass traits of finishing yearling steers and estimated diet net energy value. J Anim Sci 68, 2208-2216

Brethour JR, Sirny RJ, Tillman AJ (1958) Further studies concerning the effects of fats in sheep rations. J Anim Sci 17, 171-179

Cameron CW, Hogue DE (1968) Effect of varying dietary corn oil and hay-grain ration on lamb growth and fat characteristics. $J$ Anim Sci $27,553-556$

Chalupa W, Vecchiarelli B, Elser RE, Kronfeld DS, Schlan D, Palmquist DL (1986) Ruminal fermentation in vivo as influenced by long chain fatty acids. J Dairy Sci 69, 1293-1301

Chandler PT, Kesler EM, Mc Carthy RD, Johnston RP (1966) Additions of corn oil and soyprotein to calf diets. J Anim Sci 25, 898-899 (Abst)

Cordiez E, Lambot O, Bienfait JM, Pondant A, Van Eenaeme C (1977) La pulpe séchée de betterave sucrière : une possibilité d'économiser les céréales dans la production de viande bovine. Rev Mond Zootech 21, 18-23

Czerkawski JW, Christie WW, Breckenridge G, Hunter ML (1975) Changes in rumen metabolism of sheep given increasing amount of linseed oil in their diet. Br J Nutr 34, 25-44

Dryden FD, Marchello JA (1973) Influence of dietary fats upon carcass lipid composition in the bovine. J Anim Sci 37, 33-39

Garret WN, Yang YT, Dunkley WL, Smith LM (1976) Energy utilization, feedlot performance and fatty acid composition of beef steers fed protein encapsulated tallow or vegetable oils. J Anim Sci 42, 1522-1533

Gibney MJ, l'Estrange JL (1975) Effects of dietary unsaturated fat and of protein source on melting point and fatty acid composition of lamb fat. J Agric Sci (Camb) 84, 291-296
Harfoot CG (1981) Lipid metabolism in the rumen. In: Lipid ruminants animal (Pergamon Press). (WW Christie, ed) 21-55

Henry RJ, Cannon DC, Winckeiman JW (1974) Clinical chemistry. In: Principles an techniques. (Harper and Row, eds). New-York. 517-518, 541-553, 1289-1291

Ikwuegbu OA, Sutton JD (1982) The effect of varying the amount of linseed oil supplementation on rumen metabolism in sheep. $\mathrm{Br} \mathrm{J}$ Nutr 48, 365-374

llian MA, Razzaque MA, Salman AJ (1988) Use of fat diets of sheep in hot environments. II Effect on rumen metabolism. Anim Feed $\mathrm{Sci}$ Technol 19, 343-350

Johnson RR, Mc Clure KE (1972) High fat rations for ruminants. I. the addition of saturated and unsaturated fats to high roughage and high concentrate rations. J Anim Sci 34, 501-509

Lassiter JW (1968) Effect of dietary fat source and buffer level on composition of depot fats gains and liver manganese of sheep. J Anim Sci 27, 1466-1471

Marchello MJ, Fontenot JP, Kelly RF (1969) Effect of pre-and post-ruminal administration of corn oil on ovine fat. J Anim Sci 29, 874-881

Mehrez AZ, Ørskov ER (1977) Study of the artificial fiber bag technique for determining the digestibility of feeds in the rumen. J Agric Sci $88,645-650$

Michaux C, Stasse A, Sonnet R, Leroy P, Hanset $R$ (1983) La composition de la carcasse de taureaux culards Blanc-Bleu Belge. Ann Méd Vét 127, 349-375

Palmer DW, Peters JT (1969) Automated determination of free amino groups in serum and plasma using 2, 4, 6-trinitrobenzene sulfonate. Clin Chem 19, 891-901

Paimquist DL (1988) The feeding value of fat. In: World animal science. (Ørskov ER, ed) Elsevier, 293-311

Palmquist DL, Conrad HR (1978) High fat rations for dairy cows : effects on feed intake, milk and fat production and plasma metabolites. J Dairy Sci 61, 890-901

Roberts WK, Mc Kirdy JA (1964) Weight gains carcass fat characteristics and ration digesti- 
bility in steer affected by dietary rapeseed oil, sunflowerseed oil and animal tallow. $J$ Anim Sci 23, 682-687

Ter Meulen V, Molnar S (1975) Untersuchungen zur Morphologie und Physiologie des perirenalen Fettgewebes beim kalb und der Einfluss der Umgebungstemperatur auf seine Funktion. 2. Methodik und Versuchsergebnisse. Z Tierphysiol, Tierernähr Futtermittelkde 35, 144-163

Willey NB, Riggs JK, Colby RW, Butler OD, Reisser J, Reiser R (1952) The influence of level of fat and energy in the ration upon feedlot performance and carcass composition of fattening steers. J Anim Sci 11, 705711

Zinn RA (1988) Comparative feeding value of supplemental fats in finishing diets for feedlot steers supplemented with and without monensin. J Anim Sci 66, 213-227

Zinn RA (1989) Influence of level and source of dietary fat on its comparative feeding value in finishing diets for steers: feedlot cattle growth and performance. J Anim Sci 67, 1029-1037 